Extended Hubbard model on a $\mathrm{C}_{20}$ molecule

This article has been downloaded from IOPscience. Please scroll down to see the full text article.

2007 J. Phys.: Condens. Matter 19456206

(http://iopscience.iop.org/0953-8984/19/45/456206)

View the table of contents for this issue, or go to the journal homepage for more

Download details:

IP Address: 130.39.182.94

The article was downloaded on 22/10/2010 at 20:48

Please note that terms and conditions apply. 


\title{
Extended Hubbard model on a $\mathrm{C}_{20}$ molecule
}

\author{
Fei Lin ${ }^{1}$, Erik S Sørensen ${ }^{2}$, Catherine Kallin ${ }^{2}$ and A John Berlinsky ${ }^{2}$ \\ ${ }^{1}$ Department of Physics, University of Illinois at Urbana-Champaign, Urbana, IL 61801, USA \\ ${ }^{2}$ Department of Physics and Astronomy, McMaster University, Hamilton, ON, L8S 4M1, Canada
}

Received 27 August 2007

Published 11 October 2007

Online at stacks.iop.org/JPhysCM/19/456206

\begin{abstract}
The electronic correlations on a $\mathrm{C}_{20}$ molecule, as described by an extended Hubbard Hamiltonian with a nearest-neighbor Coulomb interaction of strength $V$, are studied using quantum Monte Carlo and exact diagonalization methods. For electron-doped $\mathrm{C}_{20}$, it is known that pair binding arising from a purely electronic mechanism is absent within the standard Hubbard model $(V=0)$. Here we show that this is also the case for hole doping for $0<U / t \leqslant 3$ and that, for both electron and hole doping, the effect of a non-zero $V$ is to work against pair binding. We also study the magnetic properties of the neutral molecule, and find transitions between spin singlet and triplet ground states for either fixed $U$ or $V$ values. In addition, spin, charge and pairing correlation functions on $\mathrm{C}_{20}$ are computed. The spin-spin and charge-charge correlations are very short-range, although a weak enhancement in the pairing correlation is observed for a distance equal to the molecular diameter.
\end{abstract}

(Some figures in this article are in colour only in the electronic version)

\section{Introduction}

Shortly after the discovery of superconductivity in $\mathrm{C}_{60}$, it was suggested by Chakravarty, Kivelson and Gelfand [1-3] that an electronic mechanism, in which pairs of electrons preferentially reside on a single molecule rather than on neighboring molecules, might provide the pairing mechanism for superconductivity. Using second-order perturbation theory they found evidence for pair binding, above a threshold value of $U / t \approx 3$. They also found that this attraction between doped electrons is accompanied by a violation of Hund's rule, which requires maximal spin, for the two-electron-doped $\mathrm{C}_{60}$, and that for $U / t>3$ the ground state for two-electron-doped $\mathrm{C}_{60}$ has spin zero [1, 2]. However, recent calculations [4], using quantum Monte Carlo (QMC) techniques, suggest that the repulsive Hubbard model does not lead to pairing on $\mathrm{C}_{60}$. On the other hand, there are geometries where pair binding is known to occur [3,5]. In particular, White et al in exact diagonalization (ED) studies of the extended Hubbard model on the much smaller $\mathrm{C}_{12}$ (truncated tetrahedron) molecule have shown that a negative pair-binding energy (effective attraction between doped electrons) exists for an intermediate value of the on-site Coulomb interaction $U$ (see equation (1) and figure 3(a)). 
A more realistic model of the fullerenes would include longer-ranged Coulomb repulsions, and it was found that this pairing energy also survives in $\mathrm{C}_{12}$ for modestly repulsive values of the nearest-neighbor (NN) interaction, $V$, but increasing $V$ eventually kills the pair binding. The same violation of Hund's rule as in $\mathrm{C}_{60}$ was also observed in $\mathrm{C}_{12}$ (see [5] and figure 3(b)).

With a different extended Hubbard model, Sondhi et al [6] studied the effects of both NN interaction $V$ and the off-diagonal interactions on the pair-binding energy and Hund's rules violation in the $\mathrm{C}_{60}$ molecule. Using perturbative calculations, they found that the NN interaction $V$ terms suppress pair binding while the off-diagonal terms enhance it. Goff and Phillips [7, 8] considered the effects of both NN interaction $V$ and longer-range terms, $V$, on the pair-binding energy, again by perturbation theory, and also found that the inclusion of $V$ terms strongly suppresses pair binding in $\mathrm{C}_{60}$.

The fact that ED studies found pair binding for the smaller $\mathrm{C}_{12}$ molecule [5] and the recent rapid development of experimental techniques for the synthesis of $C_{20}$ solid phases [9, 10] make it interesting and timely to explore correlation effects in $\mathrm{C}_{20}$, the smallest gas-phase fullerene molecule which has dodecahedral geometry [11]. In [12], we briefly reported on pair binding for electron-doped $\mathrm{C}_{20}$ for a wide range of values of $U / t \leqslant 100$, but with $V=0$, using both QMC for $U / t \leqslant 3$ and ED for the full range of values. Using cluster perturbation theory $[13,14]$ we also identified a metal-insulator transition near $U_{\mathrm{c}} / t \sim 4.2$ for molecular solids formed of $\mathrm{C}_{20}$. In this paper, we provide further details of our numerical techniques and consider both electron and hole doping for an extended Hubbard model with both on-site and $\mathrm{NN}$ repulsion. We also study density-density, spin-spin and pairing correlation functions as a function of separation on the molecule.

The extended Hubbard Hamiltonian on a single $\mathrm{C}_{20}$ molecule is defined as

$$
H=-t \sum_{\langle i j\rangle \sigma}\left(c_{i \sigma}^{\dagger} c_{j \sigma}+\text { h.c. }\right)+U \sum_{i} n_{i \uparrow} n_{i \downarrow}+V \sum_{\langle i j\rangle} n_{i} n_{j},
$$

where $c_{i \sigma}^{\dagger}\left(c_{i \sigma}\right)$ is an electron creation (annihilation) operator on site $i$, indices $i, j$ run over 20 sites of a dodecahedron, $U$ is the on-site Coulomb interaction, $V$ is the NN Coulomb interaction, and $n_{i}=n_{i \uparrow}+n_{i \downarrow}$ is the number of electrons on site $i$. Our goal here is to focus on strong correlation effects in $\mathrm{C}_{20}$ using exact numerical techniques. The Hamiltonian equation (1) is a simplified model of $\mathrm{C}_{20}$, but it still largely captures such correlation effects. We calculate ground-state energies as a function of both $U$ and $V$ for neutral, one- and twoelectron dopings. Comparisons between these energies show that the electronic pair-binding energy $\Delta_{\mathrm{b}}(21)=E(20)+E(22)-2 E(21)$ is positive (repulsive) for the parameter ranges studied $(0<U / t \leqslant 3$ for $V / t=0.2$ and $0.20 \leqslant V / t \leqslant 0.46$ for $U / t=1)$. This implies that it is energetically favorable for two electrons to stay on different molecules as opposed to the same molecule. We also find that the existence of a NN Coulomb interaction $V$ enhances this tendency, as expected, in order to reduce the intramolecular Coulomb interaction energy. For hole doping, the corresponding hole pair-binding energy $\Delta_{\mathrm{b}}(19)=E(18)+E(20)-2 E(19)$ is again positive (repulsive) for the parameter range $(0<U / t \leqslant 3$ and $V=0)$, i.e. there is an effective repulsion between two doped holes on the same $\mathrm{C}_{20}$ molecule.

Unlike the case of $\mathrm{C}_{60}$, the highest occupied molecular orbital (HOMO) of the neutral $\mathrm{C}_{20}$ molecules, in the weakly interacting limit, is a four-fold orbitally degenerate level occupied by two electrons (see figure 1). Hund's rules predict for this case that the two electrons occupy different orbitals and have total $S=1$, implying that, in the absence of a Jahn-Teller distortion, the neutral molecule has a magnetic moment. In previous work [12], for $V=0$, we have confirmed this magnetic moment for $0<U / t<3$ and shown that at the metal-insulator transition, $U_{\mathrm{c}}$, the ground state changes from a spin triplet to a singlet for neutral $\mathrm{C}_{20}$ and from $S=2$, through $S=1$, to $S=0$ for $\mathrm{C}_{20}^{2-}$. Here we extend this analysis to determine the 


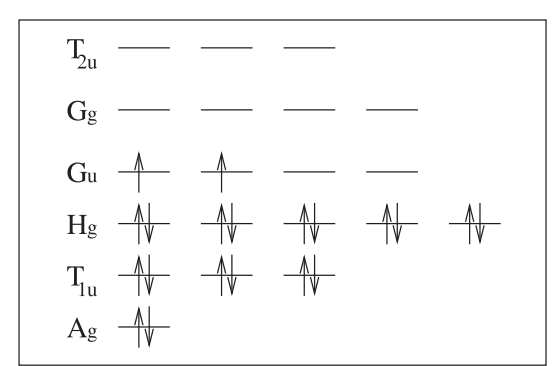

Figure 1. Huckel molecular orbitals of a neutral dodecahedral $\mathrm{C}_{20}$ molecule [15].

ground-state spin configuration for neutral $\mathrm{C}_{20}$ for a fixed value of $U / t=2$ as a function of $V$, and find a level crossing between $V / t=1$ and 1.5 for spin triplet and singlet states. For $U / t=2$, we estimate the critical $V_{\mathrm{c}} / t$ to be 1.1 for the spin triplet to singlet transition of the neutral molecule. In light of our results for $V=0$ [12], we expect that, in this case too, the magnetic transition at $V_{\mathrm{c}}$ will coincide with a metal-insulator transition for molecular solids formed of $\mathrm{C}_{20}$. We also investigate the pair-binding energy for the hole-doped case for both $V=0$ and $V \neq 0$, and examine the effect of a non-zero $V$ on Hund's rule.

The occurrence of orbital degeneracy and the resulting magnetic moment are tied to the icosahedral symmetry of the molecule. Simple molecular orbital calculations strongly suggest that the molecular symmetry is lowered by a Jahn-Teller effect from $\mathrm{I}_{\mathrm{h}}$ to $\mathrm{D}_{3 \mathrm{~d}}$, with the HOMO being a non-degenerate singlet [16]. However, the correlation effects that give rise to Hund's rule compete with this tendency to form a singlet ground state, and hence they also compete with the Jahn-Teller effect. As reported previously [12], we find that when the on-site Coulomb interaction $U / t$ is sufficiently large $(U \gtrsim 4.2 t$ ), the ground state is gapped with $S=0$ and the $\mathrm{I}_{\mathrm{h}}$ symmetry is likely stable against a $\mathrm{D}_{3 \mathrm{~d}}$ distortion. In order to focus more exclusively on the effects of the non-zero $V$ term, here we shall assume that the icosahedral symmetry is unbroken even for smaller $U$ values.

In the next section, we briefly introduce the projection quantum Monte Carlo (PQMC) [17] and ED methods for this model. This is followed, in section 3, by a comparison of PQMC with $E D$ results on a $C_{12}$ and a discussion of Hund's rule violation in $C_{12}$. In section 4 we focus on the $\mathrm{C}_{20}$ molecule. Hole pair binding in $\mathrm{C}_{20}$ is discussed and the influence of a non-zero nearestneighbor $V$ on the pair binding is investigated and results for the triplet-singlet transition with $V$ are described along with calculations of several correlation functions in the $\mathrm{C}_{20}$ molecule. Section 5 contains discussion and conclusions.

\section{Method}

\section{1. $P Q M C$}

As noted in [18], the idea in PQMC simulations of the extended Hubbard model is to decouple the two-body interaction terms (both $U$ and $V$ terms) in the partition function by means of discrete Hubbard-Stratonivich transformations [19]. The resultant one-body terms are coupled to several auxiliary Ising spin fields that live either on the lattice sites ( $U$ term) or on the lattice bonds ( $V$ term). One such discrete transformation in the $V$ term is given by

$$
\mathrm{e}^{-\Delta \tau V n_{i \alpha} n_{j \beta}}=\frac{1}{2} \operatorname{Tr}_{\left\{\sigma_{i j}^{\alpha \beta}\right\}} \mathrm{e}^{\lambda_{2} \sigma_{i j}^{\alpha \beta}\left(n_{i \alpha}-n_{j \beta}\right)-\frac{\Delta \tau V}{2}\left(n_{i \alpha}+n_{j \beta}\right)}
$$

where $\alpha, \beta=\uparrow, \downarrow, \sigma_{i j}^{\alpha \beta}= \pm 1$ is the auxiliary Ising spin on bond $(i, j), \Delta \tau$ is the discrete 
imaginary time slice in PQMC, and the parameter $\lambda_{2}$ is determined by $\tanh ^{2}\left(\lambda_{2} / 2\right)=$ $\tanh \left(\frac{\Delta \tau V}{4}\right)$. The same decoupling equation applies for on-site Coulomb interactions, i.e. $i=j$, except that the constant $V$ is replaced by $U$ and $\lambda_{2}$ by $\lambda_{1}$, which is similarly given by $\tanh ^{2}\left(\lambda_{1} / 2\right)=\tanh \left(\frac{\Delta \tau U}{4}\right)$. These one-body fermionic terms in the partition function can then be traced out explicitly, leaving traces over the auxiliary Ising spins, which can be evaluated by Monte Carlo (MC) [19]:

$$
\begin{aligned}
Z & =\sum_{\{\sigma\}} \prod_{\alpha} \operatorname{det}\left[1+B_{L}(\alpha) B_{L-1}(\alpha) \cdots B_{1}(\alpha)\right] \\
& =\sum_{\{\sigma\}} \operatorname{det} \mathrm{O}(\{\sigma\})_{\uparrow} \operatorname{det} \mathrm{O}(\{\sigma\})_{\downarrow},
\end{aligned}
$$

where $\{\sigma\}=\left\{\sigma^{1}, \sigma^{2}, \sigma^{3}, \sigma^{4}, \sigma^{5}\right\}$ is the set of five species of Ising fields, with $\sigma^{1}$ representing the on-site Ising spins and $\sigma^{2-5}$ the $\mathrm{NN}$ bond Ising spins (one for each of the four spin configurations). The $B_{l}$ matrices are defined as

$$
\begin{aligned}
& B_{l}(\alpha)=\mathrm{e}^{-\Delta \tau K / 2} \mathrm{e}^{W^{\alpha}(l)} \mathrm{e}^{-\Delta \tau K / 2}, \\
& (K)_{i j}= \begin{cases}-t & \text { for } i, j \mathrm{NN}, \\
0 & \text { otherwise, }\end{cases} \\
& W_{i j}^{\alpha}(l)=\alpha\left[\delta_{i j} \lambda_{1} \sigma_{i}^{1}(l)+\delta_{\langle i j\rangle} \lambda_{2} \sum_{m=2}^{5} \sigma_{i j}^{m}\right], \\
& \delta_{\langle i j\rangle}= \begin{cases}1 & \text { for } i, j \mathrm{NN}, \\
0 & \text { otherwise, }\end{cases}
\end{aligned}
$$

where $l=1, \ldots, L$ is the time slice index, and $\alpha= \pm 1$ denotes the two determinants in equation (3).

A complete MC sweep through the lattice will therefore consist of trial flipping of one species of auxiliary Ising spins on all the lattice sites and trial flipping of four species of auxiliary Ising spins on all the NN bonds in the lattice system. Fast calculation of the probability ratio in flipping one bond Ising spin at one time slice is still possible using the local update technique [20], except that one needs to apply the probability ratio formula twice for each bond Ising spin flip (which affects two sites).

We remark that in this decomposition scheme it is possible to treat even longer-range Coulomb interactions (e.g. next-nearest-neighbor (NNN) Coulomb interactions, etc) by introducing more species of auxiliary Ising spins that live on these longer bonds. The only problem is that one needs to walk through a larger and larger phase space of the auxiliary Ising spins during the MC simulations, which will, of course, increase the computation time. Practically, we find that, to collect the same amount of data, the CPU time doubles for $V \neq 0$ compared with the $V=0$ case.

In a typical calculation the projection factor $\beta$ in PQMC was taken to be $\beta=10 / t$, and the discrete time slice was set at $\Delta \tau=0.05 / t$. Typically, $10^{3} \mathrm{MC}$ warm-up sweeps through the whole space-time lattice are performed before collecting data. To estimate the statistical errors, we use the same method as was used in [4].

\subsection{Exact diagonalizations (ED)}

The exact diagonalizations on $\mathrm{C}_{12}$ are performed using standard Lanczos techniques and we therefore focus on the ED of $\mathrm{C}_{20}$. We always use the total particle number $N$ and total $S_{z}$ as quantum numbers, since they are conserved and we perform ED in the corresponding reduced 


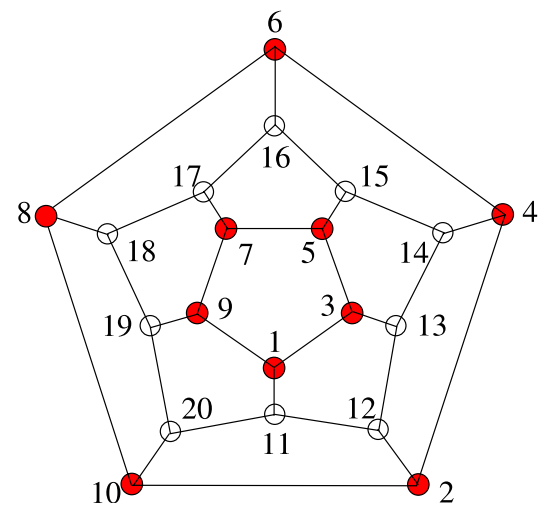

Figure 2. Dodecahedral $\mathrm{C}_{20}$ geometry in two-dimensional (2D) view. Solid and empty points denote two sets (orbits) of carbon atoms divided by the $\mathrm{S}_{10}$ symmetry.

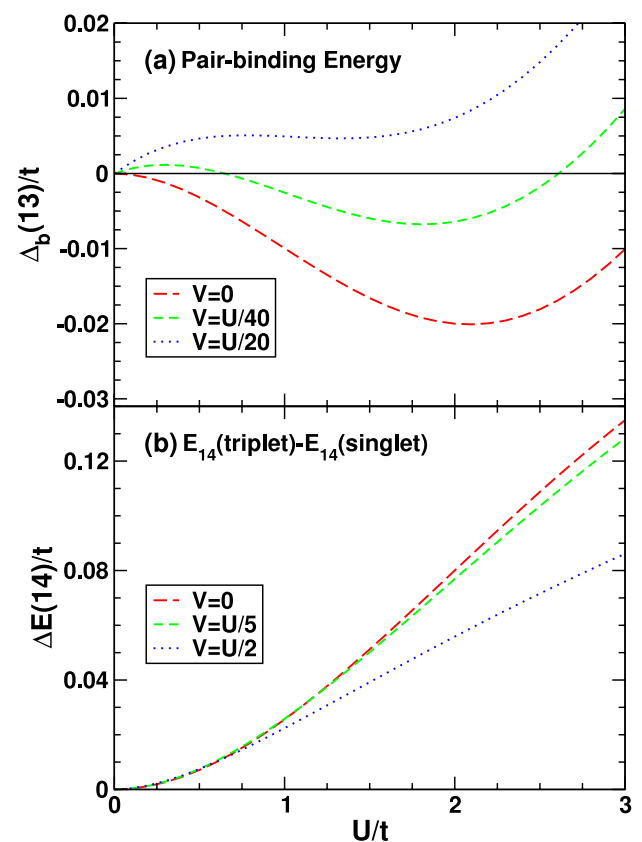

Figure 3. (a) Variation of the pair-binding energy $\Delta_{\mathrm{b}}(13)=E(12)+E(14)-2 E(13)$ of a truncated tetrahedron molecule $\left(\mathrm{C}_{12}\right)$ with $U$ and $V$ as in figure 3 in [5]. (b) Hund's rules violation in the two-electron-doped $\mathrm{C}_{12}$ molecule, where $\Delta E(14)=E_{14}$ (triplet) $-E_{14}$ (singlet).

Hilbert space. In addition, ED are performed using the $S_{10}$ sub-group symmetry present in the point group $\mathrm{I}_{\mathrm{h}}$. The improper rotations generated by the elements of $S_{10}$ can be visualized as a rotation of an angle $2 \pi / 10$ around the center of a pentagon followed by a reflection in a plane perpendicular to the rotation axis. This is illustrated in figure 2 where the numbering of the sites is to be understood in the following way: the sites 1 through 10 are shifted up by 1 (modulo 10) under $S_{10}$ and the sites 11-20 are shifted in a similar manner. Hence, under the action of the $S_{10}$ group, two different orbits exist, marked by the solid and open points in figure 2. Many other symmetries exist but the $S_{10}$ symmetry is large and relatively easy to implement, and we have 
not exploited additional symmetries since the added CPU time needed to implement them was significant enough to offset the time gained from reducing the size of the Hilbert space. The $S_{10}$ quantum number can be thought of as a pseudo angular momentum, $j_{10}$, and for each value of $N$ and $S_{z}$ we have to find the value of $j_{10}$ that corresponds to the ground state. In many cases it is not an obvious value and it is often non-zero. In the accompanying tables we show the values of $j_{10}$ corresponding to the listed energies, and in table 4 we show complete dispersion of the lowest magnetic modes for neutral $\mathrm{C}_{20}$ as a function of $j_{10}$.

The calculations are fully parallelized Lanczos calculations executed on SHARCNET computers. A typical calculation performed at half-filling for $N=20, S z=0$ that, after $S_{10}$ symmetry reductions, requires a Hilbert space of $\mathcal{N}=3418725024$, is performed with $P=64$ CPUs using about $540 \mathrm{~s}$ of CPU time (for each CPU) per Lanczos iteration. The memory requirement for this example is roughly $2.1 \mathrm{~Gb}$ per CPU. Excellent convergence is always observed with less than 300 Lanczos iterations, typically less than 200.

The heart of the Lanczos calculation is the matrix vector multiplication that, in this case, has to be implemented in parallel. As one of several choices, we have chosen to have each CPU apply the full matrix to one section of the vector, with each CPU returning the corresponding section of the resulting vector. The partial results from each CPU therefore need to be communicated between all $P$ processors, with each processor communicating to all the others. Due to the size of the involved Lanczos vectors $(40-60 \mathrm{~Gb})$ which greatly exceeds the available per-CPU memory, it is necessary to repeat this $P \times P$ communication step many thousands of times per Lanczos step. The communication step therefore quickly becomes the bottle-neck in the calculation unless it can be performed very efficiently. Fortunately, this is possible using non-blocking communications where the individual CPUs do not wait for a communication to complete. The drawback of using non-blocking communications is that buffer space has to be allocated until it has explicitly been verified that the communication has been completed. We have implemented a dual buffer strategy, yielding an extremely efficient communication step. The CPU time spent per CPU is, for all accessible numbers of processors that we have been able to check, overwhelmingly dominated by actual calculations rather than communications. For a fixed $\mathcal{N}$ we have then observed almost linear scaling for $P=64,128,256,384$ and 512 . The great advantage of this approach is that the complexity of Lanczos calculations scales with the size of the Hilbert space, $\mathcal{N}$, as $\mathcal{N} \log \mathcal{N}$. Neglecting the logarithm, a doubling of the size of the Hilbert space, $\mathcal{N}$, can then be almost compensated by doubling the number of processors $P$.

\section{Results for $\mathrm{C}_{12}$}

Before turning our attention to the $\mathrm{C}_{20}$ molecule we investigate the simpler $\mathrm{C}_{12}$ molecule in the truncated tetrahedron configuration. As mentioned above, previous studies [5] have found a negative pair-binding energy on this molecule that, however, became positive (repulsive interaction) in the presence of a sufficiently large $V$. The purpose of this investigation is twofold. First of all, we want to verify the correctness of our numerical approach while at the same time highlighting some of the subtleties of interpreting the PQMC data. Secondly, due to the relative ease with which calculations can be performed on this molecule, it allows for a rather detailed study of the correlation between the negative pair-binding energy and a violation of Hund's rule for the two-electron-doped molecule [1, 2].

\subsection{Tests on the $C_{12}$ molecule}

To test our ED program, we use the same parameters as in [5] and we are able to reproduce the same pair-binding energy as shown in figure 3(a). In table 1, we see good agreement between 
Table 1. Comparison of ED and PQMC calculations on the truncated tetrahedron (12 sites) at $U=2 t$ and $V=0.2 t . E_{n}\left(S_{z}\right)$ is the energy of a system with $n$ electrons and a $z$-component of total spin $S_{z} . \Delta_{n, m}$ is the energy difference $E_{12+n}\left(S_{z}^{n}\right)-E_{12+m}\left(S_{z}^{m}\right)$ with $\left(S_{z}^{n}, S_{z}^{m}\right)$ given in the second column. For binding energies $\Delta_{\mathrm{b}}(n)$, the second column shows $\left(S_{z}^{n+1}, S_{z}^{n-1}, S_{z}^{n}\right)$ 一 the $S_{z}$ values for the three states involved in its calculation [4].

\begin{tabular}{llllll}
\hline & $S$ & $S_{z}$ & ED & PQMC & Sign \\
\hline$E_{12}$ & 0 & 0 & -9.4647669965 & $-9.466(2)$ & 0.97 \\
$E_{13}$ & $1 / 2$ & $1 / 2$ & -6.8287003500 & $-6.829(4)$ & 0.33 \\
$E_{13}$ & $3 / 2$ & $3 / 2$ & -6.0844214907 & $-6.059(6)$ & 0.20 \\
$E_{14}$ & 0 & 0 & -4.1568425864 & $-4.11(1)$ & 0.11 \\
$E_{14}$ & 1 & 1 & -4.0772924523 & $-4.080(5)$ & 0.34 \\
$\Delta_{1,0}$ & $(1 / 2,0)$ & 2.6360666465 & $2.637(4)$ & \\
$\Delta_{1,0}$ & $(3 / 2,0)$ & 3.3803455058 & $3.407(6)$ & \\
$\Delta_{\mathrm{b}}(13)$ & $(0,0,1 / 2)$ & 0.0357911171 & $0.08(1)$ & \\
\hline
\end{tabular}

PQMC and ED energy values within statistical error bounds. An exception is found for $E_{14}$ and $S_{z}=0$, where the PQMC result lies a bit higher than the ED energy value. This is due to the mixture of singlet and triplet components in the $S_{z}=0$ sector and the near degeneracy of these two states that makes the projection of the singlet ground state out of the mixed state difficult [4]. We will see that this difficulty does not occur for $\mathrm{C}_{20}$, where the ground state with two-electron doping is in the spin-2 sector for $U / t \leqslant 3$. Hence the pair-binding energy extracted for $\mathrm{C}_{20}$ by PQMC for $U / t \leqslant 3$ is more reliable than the one for $\mathrm{C}_{12}$.

\subsection{Hund's rule violation for $C_{12}^{2-}$}

In the perturbation theory studies of pair binding in the larger fullerene $C_{60}[1,2]$, it was noted that a negative pair-binding energy (effective attraction) was correlated with a violation of Hund's rule for the two-electron-doped molecule; i.e. that for $\mathrm{C}_{60}^{2-}$ the ground state was found to be a singlet. Although our QMC results did not support the existence of pair binding in $\mathrm{C}_{60}^{2-}$ and found a spin triplet ground state, it is of interest to examine the correlation between pair binding and the violation of Hund's rule in $\mathrm{C}_{12}$. The non-interacting $V=U=0$ neutral molecule has completely filled levels and hence a total spin zero. Added electrons therefore enter an unfilled level with an orbital degeneracy of three. Hund's rule would then predict $\mathrm{C}_{12}^{2-}$ to have total $S=1$. What we find is that the ground state of $\mathrm{C}_{12}^{2-}$ is a singlet both when the pairing is attractive and when it is driven repulsive by increasing the nearest-neighbor repulsion $V$. This is shown in figure 3(b), where the singlet state is found to lie below the triplet state for both positive and negative pair-binding energies, for the range of $U$ and $V$ studied. Thus, for this case, Hund's rule is found to be violated where pair-binding occurs as well as where it does not.

\section{Results for $\mathrm{C}_{20}$ molecule}

We now turn to the more interesting case of the $\mathrm{C}_{20}$ molecule. Compared with the $V=0$ case, where PQMC already has a sign problem for the non-bipartite dodecahedral molecular geometry, the NN Coulomb interaction $V$ terms introduce more sources of negative probability weight, lowering the average value of the sign. Figure 4 shows the average sign for these two cases. For the worst case $\left(N=20, S_{z}=1, U / t=3, V / t=0.2\right)$, where the average sign is as low as 0.05 , we have collected $7.2 \times 10^{7} \mathrm{MC}$ lattice sweeps. This gives a relatively large but nevertheless meaningful error bar (see figure 5(a)). For other parameter values, we have 


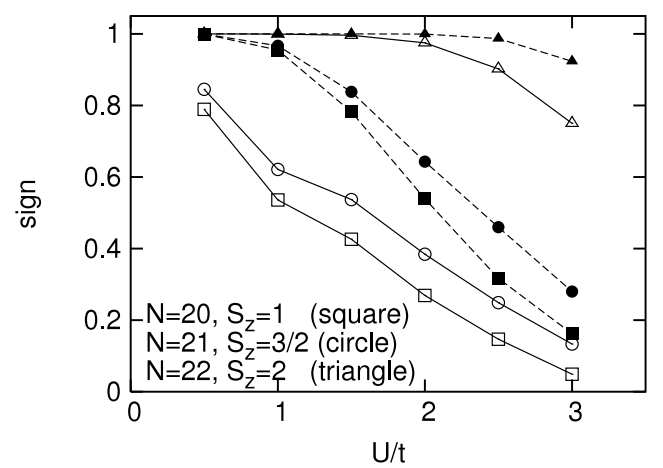

Figure 4. Average sign behavior for both $V / t=0$ (solid symbols) and $V / t=0.2$ (hollow symbols) at different fillings $N=20,21,22$. The lines connecting the points are guides to the eye only.
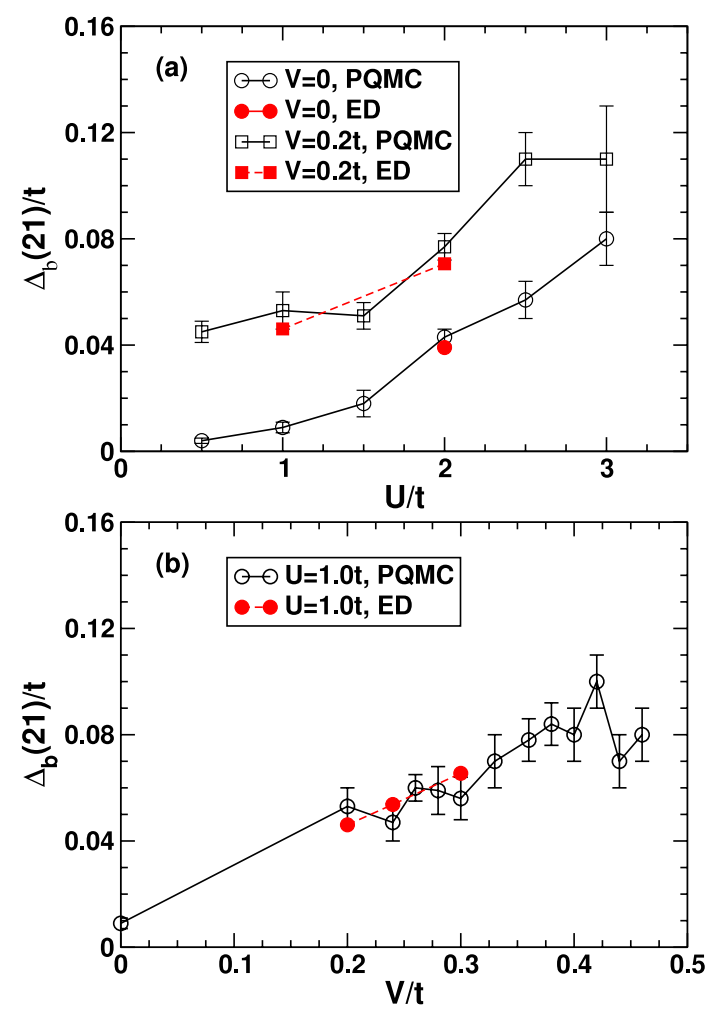

Figure 5. Electronic pair-binding energies $\Delta_{\mathrm{b}}(21) / t$ as a function of $U / t$ and $V / t$ from ED and PQMC simulations. (a) The variation of pair-binding energy with $U / t$ for fixed $V / t$ values. (b) The variation of pair-binding energy with $V / t$ for fixed $U / t=1$. The lines connecting MC and ED points are guides to the eye only.

collected about $2.2 \times 10^{7} \mathrm{MC}$ sweeps. The acceptance ratio for the on-site Ising spin trial flipping ranges from $80 \%(U / t=3)$ to $93 \%(U / t=1)$, while that for the bond Ising spins is about $95 \%$ due to the small value of $V / t=0.2$. 
Table 2. Comparison of ground-state energies from ED and PQMC calculations on the $\mathrm{C}_{20}$ molecule at $U / t=2$ and $V=0$. See the caption in table 1 for the corresponding definition of various quantities.

\begin{tabular}{lllcccc}
\hline & $S$ & $S_{z}$ & ED & $j_{10}$ & PQMC & Sign \\
\hline$E_{18}$ & 0 & 0 & -22.4044466933 & 0 & $-22.402(1)$ & 1.00 \\
$E_{18}$ & 1 & 1 & -21.6778357505 & $\pm 3,5$ & $-21.637(2)$ & 0.49 \\
$E_{19}$ & $1 / 2$ & $1 / 2$ & -21.5223243600 & $\pm 1, \pm 3$ & $-21.5227(6)$ & 0.64 \\
$E_{19}$ & $3 / 2$ & $3 / 2$ & -20.8990191757 & $0, \pm 4$ & $-20.826(3)$ & 0.35 \\
$E_{20}$ & 1 & 0 & -20.5983834340 & $0, \pm 2$ & $-20.533(3)$ & 0.26 \\
$E_{20}$ & 1 & 1 & -20.5983834340 & $0, \pm 2$ & $-20.597(2)$ & 0.54 \\
$E_{20}$ & 0 & 0 & -20.5920234654 & $0, \pm 2, \pm 4$ & & \\
$E_{20}$ & 2 & 2 & -19.9634427212 & $\pm 2, \pm 4,5$ & & \\
$E_{21}$ & $3 / 2$ & $1 / 2$ & -19.6331786587 & $\pm 1, \pm 3$ & $-19.465(8)$ & 0.19 \\
$E_{21}$ & $3 / 2$ & $3 / 2$ & -19.6331786587 & $\pm 1, \pm 3$ & $-19.634(1)$ & 0.64 \\
$E_{22}$ & 2 & 0 & -18.6289129089 & 0 & $-18.282(7)$ & 0.10 \\
$E_{22}$ & 2 & 1 & -18.6289129089 & 0 & $-18.448(5)$ & 0.32 \\
$E_{22}$ & 2 & 2 & -18.6289129089 & 0 & $-18.628(1)$ & 1.00 \\
\hline
\end{tabular}

\subsection{Pair-binding energy}

Table 2 shows the energies of the $\mathrm{C}_{20}$ molecule at different fillings from PQMC and ED for $U / t=2$ and $V=0$. Both ED and PQMC predict the ground states to be in the same spin sectors for the molecule, and the calculated energies are in agreement within MC error bounds.

In order to understand the comparison of PQMC and ED data in table 2, it is important to recognize a systematic weakness of PQMC which is that, when the ground state is a spin multiplet, the different partners appear to have different energies, increasing with decreasing values of $\left|S_{z}\right|$, because the states with smaller values of $\left|S_{z}\right|$ mix with higher-lying states that have the same value of $\left|S_{z}\right|$. In general, except for statistical error, a state with $S_{z}=0$ will appear to lie above its partners with the same total $S$. This tendency is apparent in the results for $E_{20}(S=1), E_{21}(S=3 / 2)$, and $E_{22}(S=2)$. Conversely, if a ground state with $S_{z}=0$ lies below a state with $S_{z}=1$, we expect the ground state to be a singlet. However, in this case, the value of the ground-state energy will be perturbed upward by any admixture of the next higher state with $S=1, S_{z}=0$, as happened for $E_{14}(S=0)$ in table 1 . In general, it is also true that accurate PQMC results are more easily obtained when the average sign is close to 1 compared to when the average sign is small.

Pair-binding energies $\Delta_{\mathrm{b}}(21) / t$ (electron) and $\Delta_{\mathrm{b}}(19) / t$ (hole) as a function of both $U / t$ and $V / t$ are shown in figure 5 and 6 , respectively. For $V=0$, for both electron and hole doping, we see that the pair-binding energy is always positive (repulsive) for $U / t>0$, and increases with increasing $U / t$. This is the same behavior as we observed for the $\mathrm{C}_{60}$ molecule [4]. Turning on the NN Coulomb interaction $V(V / t=0.2$ in figure 5(a)) increases the pair-binding energy further. Hence, putting two extra electrons on the same neutral molecule becomes more costly when the NN Coulomb interaction is not negligible. Panel (b) in figure 5 shows the variation of the pair-binding energy as a function of $V / t$ for fixed $U / t=1$. Again the pair-binding energy is positive (repulsive), and generally increases with $V / t$. The agreement between ED and PQMC results is fairly good and, even though the PQMC data show some tendency to non-monotonic behavior for this interval of $V / t$, the ED results show that this is explained by the natural statistical spread of the data. Hence, in the regime $V<V_{\mathrm{c}}, U<U_{\mathrm{c}}$, the pair-binding energy increases with both $U$ and $V$ and, energetically, it becomes increasingly 


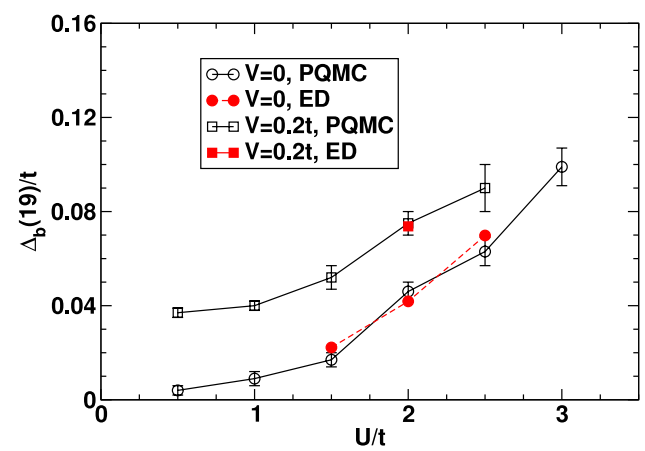

Figure 6. Hole pair-binding energies $\Delta_{\mathrm{b}}(19) / t$ as a function of $U / t$ for $V=0$ from ED and PQMC simulations. The lines connecting MC points are guides to the eye only.

Table 3. Ground-state energies for neutral, one- and two-electron-doped $\mathrm{C}_{20}$ molecules at $U / t=3,5$ and $V=0$ from PQMC and ED, which shows a transition between Hund's and antiHund's states at $3<U / t<5$ for neutral, one-, and two-electron-doped molecules, respectively. Data without error bars are from ED.

\begin{tabular}{llllllll}
\hline & $S$ & $S_{z}$ & $U / t=3$ & $S$ & $S_{z}$ & $U / t=5(\mathrm{ED})$ & $j_{10}$ \\
\hline$E_{20}$ & 1 & 0 & $-17.04(2)$ & 0 & 0 & -12.111284292 & 5 \\
$E_{20}$ & 1 & 1 & $-17.036(6)$ & 1 & 1 & -11.877033283 & $0, \pm 2$ \\
$E_{21}$ & $3 / 2$ & $1 / 2$ & $-15.29(6)$ & $1 / 2$ & $1 / 2$ & -9.1165560273 & $\pm 1, \pm 3,5$ \\
$E_{21}$ & $3 / 2$ & $3 / 2$ & $-15.529(5)$ & $3 / 2$ & $3 / 2$ & -8.9633623599 & $\pm 1, \pm 3$ \\
$E_{22}$ & 2 & 0 & -13.936353 & 1 & 0 & -5.9715313615 & $\pm 2, \pm 4$ \\
$E_{22}$ & 2 & 1 & $-13.81(2)$ & 1 & 1 & -5.9715313615 & $\pm 2, \pm 4$ \\
$E_{22}$ & 2 & 2 & $-13.935(1)$ & & & & \\
\hline
\end{tabular}

favorable for two electrons to stay on two different $\mathrm{C}_{20}$ molecules. However, we note that, for $V=0$ and $U>U_{\mathrm{c}}$, it was previously found [12] that the pair-binding energy decreases with $U$, reaching a minimum at $U / t \sim 10$, before increasing and reaching a finite value in the $U \rightarrow \infty$ limit.

\subsection{Hund's rule}

It is also clear, from the data in tables 2 and 3, that Hund's rule is obeyed for the corresponding range of parameters, i.e. $U / t \leqslant 3, V=0$. That is, the ground state for 20 through 22 all have the maximum values of total spin for electrons outside the $\mathrm{C}_{20}^{2+}$ core, ranging from total spin 1 for 20 electrons through total spin 2 for 22 electrons. This behavior occurs in the range of parameters where PQMC converges (for maximal $\left|S_{z}\right|$ as discussed above). As $U / t$ is increased above three, the sign problem prevents reliable PQMC calculations. This difficulty does not arise in ED where accurate calculations are possible for essentially any value of $U / t$. We have used ED to explore what happens for larger values of $U / t$ [12]. For example, results for $U=5 t$ are shown in the right-hand columns of table 3. Here Hund's rule is clearly violated. For 20 electrons, the ground state has spin zero; for 21 electrons the ground state has spin 1/2; while for 22 electrons the ground state has spin 1 . Clearly there are level crossings in the range $3<U / t<5$. Additional results in this regime are given in [12]. ED also allows the calculation of the spin gap, the gap between the ground state and the lowest-lying excited state with different total spin. Results are shown in table 4 for a neutral $\mathrm{C}_{20}$ molecule with $U=2,5,8$ and 
Table 4. ED results for the dispersion of the lowest singlet and triplet states with $j_{10}$ for neutral $\mathrm{C}_{20}$ with $U / t=2,5$ and 8 in all cases with $V / t=0$.

\begin{tabular}{|c|c|c|c|c|c|c|c|}
\hline$U$ & $S$ & $j_{10}=0$ & $j_{10}= \pm 1$ & $j_{10}= \pm 2$ & $j_{10}= \pm 3$ & $j_{10}= \pm 4$ & $j_{10}=5$ \\
\hline & 0 & -20.5920234655 & $>-19.90$ & -20.5920234655 & $>-19.90$ & -20.5920234655 & -20.0527029539 \\
\hline \multirow[t]{2}{*}{2} & 1 & -20.5983834340 & -19.9776970001 & -20.5983834340 & -19.9776970001 & -20.5981592741 & -19.9634427213 \\
\hline & 0 & -12.0123014488 & -11.6726562451 & -12.0123014488 & -11.6726562451 & -12.0123014488 & -12.1112842959 \\
\hline \multirow[t]{2}{*}{5} & 1 & -11.8770332831 & -11.8472120431 & -11.8770332831 & -11.8472120431 & -11.8103044760 & -11.8118179567 \\
\hline & 0 & -8.0452584717 & -7.806831 & -8.0452584717 & -7.8068365859 & -8.0452584717 & -8.1803385740 \\
\hline 8 & 1 & -7.9497836200 & -7.9415479844 & -7.9497836200 & -7.9415479844 & -7.8490047592 & -7.9156714009 \\
\hline
\end{tabular}

$V=0$. When the metal-insulator transition occurs in the vicinity of $U_{\mathrm{c}} / t \sim 4.2$, the groundstate spin changes from an orbitally degenerate $S=1$ for $U<U_{\mathrm{c}}$ to a non-degenerate singlet for $U>U_{\mathrm{c}}$. From the results presented in table 4 we see that it is the singlet state at $j_{10}=5$ that moves toward the bottom of the spectrum with increasing $U$ and, eventually, for $U>U_{\mathrm{c}}$ becomes the ground state. Focusing on the case $V / t=0$, we see from table 4 that, at $U / t=2$, the ground-state energy is a singlet $E^{1} / t=-20.5983834340$ with a gap to the lowest-lying singlet of $\Delta E^{1,0} / t=0.0063599685$. Here the superscripts denote the spin of the ground and excited states, respectively. For $U / t \geqslant 5$ we find that the ground state for the $\mathrm{I}_{\mathrm{h}}$ configuration is now a non-degenerate singlet, $S=0$, with energy $E^{0} / t=-12.1112842922$. The lowestlying triplet excitation with $\Delta E^{0,1} / t=0.2342510092$. This picture continues to hold for larger $U / t$ with the triplet gap at $U / t=8$ only slightly larger, $\Delta E^{0,1} / t=0.2305549540$.

Next we explore the ground-state spin of the neutral molecule with different $V / t$ values for a fixed $U / t=2$. Using ED techniques we determine that the ground state for $V / t=1$ and 1.5 in both cases occur for $j_{10}=0$. However, the ground state changes from a spin triplet for $V / t=1$ to a spin singlet for $V / t=1.5$. Specifically, at $V / t=1$ we find $E / t$ (singlet) $=5.702018$ and $E / t$ (triplet) $=5.639496$, whereas for $V / t=1.5$ we find $E / t($ singlet $)=17.318536$ and $E / t$ (triplet $)=17.499741$. By assuming a linear dependence of the energy on $V / t$ in this region, we determine that the level crossing occurs near $V_{\mathrm{c}} / t \sim 1.1$ for $U / t=2$.

\subsection{Correlation functions}

We have also investigated what other correlations might be induced in the $\mathrm{C}_{20}$ molecule by calculating the following correlation functions: charge-charge, spin-spin, and pairing correlations as a function of lattice distance. Similar calculations for the $\mathrm{C}_{60}$ molecule have been reported in [21] and [22].

We define the correlation functions with respect to lattice site 1 in the neutral molecule: $\left\langle n_{1} n_{i}\right\rangle$ is the charge-charge correlation, $\left\langle S_{1} \cdot S_{i}\right\rangle$ is the spin-spin correlation, and $\left\langle c_{1 \sigma}^{\dagger} c_{i,-\sigma}^{\dagger} c_{i,-\sigma} c_{1, \sigma}\right\rangle$ is the pairing correlation, where $i=1, \ldots, 20$. In figure 7 , we show the variation of these correlations for $U=2 t$ and $3 t$ as a function of lattice spacing $d_{1 i} / R$, where $d_{1 i}$ is the distance between site 1 and $i$, and $R$ is the molecular diameter. For the dodecahedral geometry, there are only five inequivalent neighbors, all at distinct distances. One can understand the on-site correlations in terms of the probabilities, $p_{n}, n=0,1,2$, for having $n$ electrons on each site. Then, the on-site correlations functions are $\left\langle n_{1}^{2}\right\rangle=p_{1}+4 p_{2}$, $\left\langle S_{1}^{2}\right\rangle=3 p_{1} / 4$, and $\left\langle c_{1 \sigma}^{\dagger} c_{1,-\sigma}^{\dagger} c_{1,-\sigma} c_{1, \sigma}\right\rangle=p_{2}$.

In figure 7 (a) we show results for the charge-charge correlation function for two different values of $U$ with $V=0$. As expected, the on-site charge-charge correlation is reduced by an increase in the on-site Coulomb interaction $U$ (panel (a)). At larger distances, the charge on 


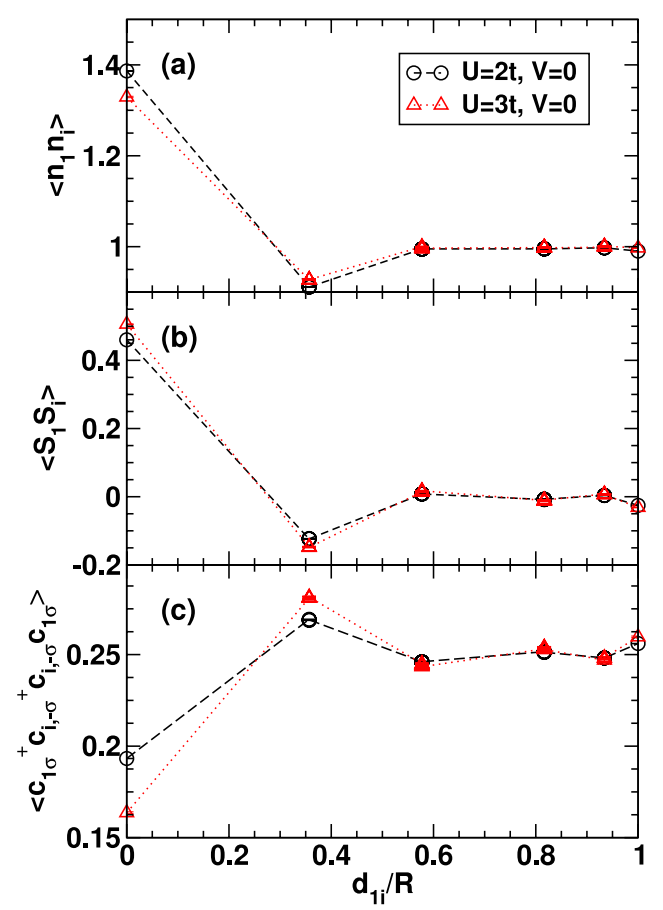

Figure 7. Variation of (a) charge-charge, (b) spin-spin, and (c) pairing correlation functions for $U=2 t, 3 t$ and $V=0$ for a $\mathrm{C}_{20}$ molecule with respect to the lattice site spacing. $d_{1 i}$ is the distance between site 1 and $i . R$ is the diameter of the $\mathrm{C}_{20}$ molecule.

site 1 and $i$ are uncorrelated. The unit value of the charge-charge correlation corresponds to uniform distribution of charge.

Figure 7(b) shows the spin-spin correlation function again for $U / t=2,3$ with $V=0$. The NN spin-spin correlation has a negative finite value, and its magnitude is enhanced by a larger $U$ value. For spatial distances larger than $\mathrm{NN}$ spacing we see that this correlation function quickly approaches 0 . Similar behavior has been observed for the spin-spin correlation in the $\mathrm{C}_{60}$ molecule in [21] and it was suggested that the rapid decay of the spin-spin correlation function was indicative of a resonant valence bond (RVB) or 'spin dimer' state. The similarity between our results and those of [21] suggest that the spin correlations in the ground state of $\mathrm{C}_{20}$ also might be described by considering valence bond states, including only dimers of relatively short length.

QMC results for the pair correlation are shown in figure 7(c) with $U / t=2,3$ and $V=0$. Interestingly, there is a peak in the pairing order when site 1 and $i$ are $\mathrm{NN}$ sites. This again supports the RVB or 'spin dimer' model for the ground state. Beyond the nearest-neighbor distance, the pairing correlation function, along with the other correlation functions, is very close to its uncorrelated value except for $d_{1 i}=R$, where the pairing order parameter is slightly enhanced. At the same time the spin-spin correlation is slightly negative, showing an antiferromagnet correlation. This corresponds to the 'dumb bell' model proposed in [22] where electron pairs are formed at the maximal distances of the molecular diameter. We note that, in the present case, the enhancement of the correlations at the distance of $R$ corresponding to this 'dumb bell' pairing is relatively weak.

We have also studied the influence of a non-zero $V$ on the correlations. In figure 8 we show results for a fixed $U / t=3$ and three different values of $V=0,0.1 t, 0.2 t$. Clearly, the 


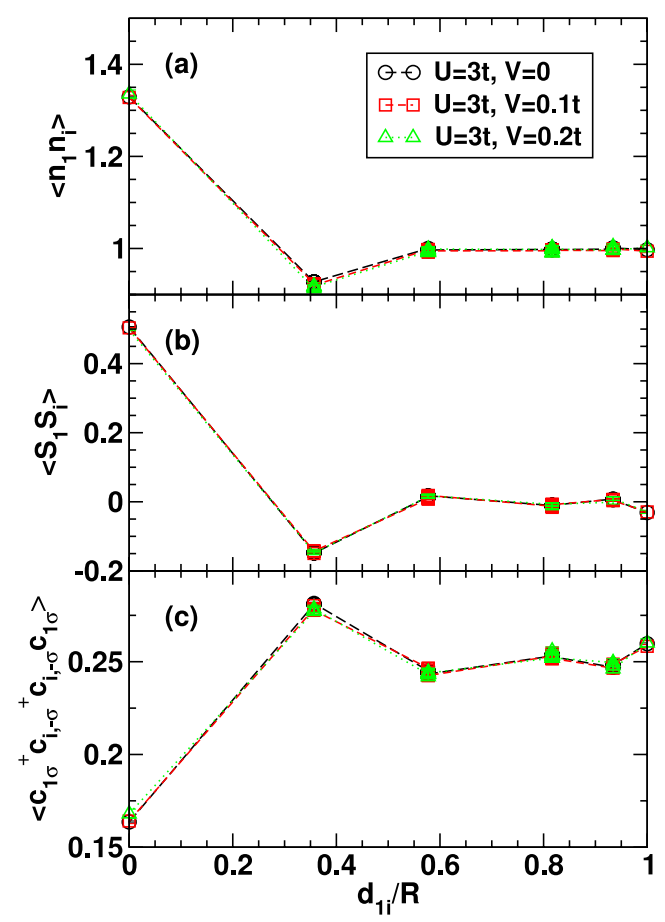

Figure 8. Variation of (a) charge-charge, (b) spin-spin, and (c) pairing correlation functions for $U=3 t$ and $V=0,0.1 t, 0.2 t$ for a $\mathrm{C}_{20}$ molecule with respect to the lattice site spacing.

effect of the NN Coulomb interaction $V$ on these correlation functions is relatively weak, with the curves being almost identical for the range of $V$ considered here.

\section{Conclusions}

In this paper we have studied the extended Hubbard model on a $\mathrm{C}_{20}$ molecule through ED and PQMC simulations. The comparison clearly elucidates the relative strengths of the two methods. PQMC is possible for much larger systems than can be treated by ED. However, ED has been applied successfully to the Hubbard model on 20 sites with 18-22 electrons, by making effective use of the capabilities of a large number of coupled processors. PQMC works best when the ground state is well separated from excited states with the same value of $S_{z}$. As a result, ground states with larger total spin $S$ and maximal $\left|S_{z}\right|$ are determined most accurately, while ground states with $S=0$ are sometimes problematic. This behavior was also found in our earlier work on $\mathrm{C}_{60}$ [4], and the comparison of $\mathrm{ED}$ and $\mathrm{PQMC}$ results for $\mathrm{C}_{20}$ is consistent with and lends confidence to those earlier results.

The pair-binding energy for $\mathrm{C}_{20}$ shows that extra added electrons (holes) prefer to sit on different molecules, rather than to reside in pairs on molecules. This rules out the possibility that the extended Hubbard model on a single $\mathrm{C}_{20}$ molecule can produce an effective attraction between electrons (holes) from purely electronic interactions. Our earlier work showed that this conclusion applies to the $\mathrm{C}_{60}$ molecule as well [4]. We also find that Hund's rule is obeyed for $U / t \leqslant 3$ and small values of $V$ and that larger values of $U$ and $V$ lead to level crossings and ground states for which Hund's rule is violated. For fixed $V=0$, we have determined that this transition happens between $U / t=3$ and 5, at $U_{\mathrm{c}} / t \sim 4.2$. And for fixed $U / t=2$, 
as a function of $V$, we have determined that this transition happens between $V / t=1$ and 1.5, at $V_{\mathrm{c}} \sim 1.1$. As was the case at the transition occurring at $U_{\mathrm{c}} / t \sim 4.2$ for $V=0$, we expect this transition to coincide with a metal-insulator transition for molecular solids formed of $\mathrm{C}_{20}$. More generally, for $U / t \leqslant 3$ and $V / t \leqslant 0.2$, we find that the spin, charge and pairing correlations fall off rapidly even in the presence of NN Coulomb repulsion. It is an interesting open question if molecular solids formed of $\mathrm{C}_{20}$, in particular away from half-filling, would display non-trivial order for $V>V_{\mathrm{c}}$. The answer to this question would be numerically demanding and we have therefore left it for future work.

\section{Acknowledgments}

This project was supported by the Natural Sciences and Engineering Research Council of Canada, the Canadian Institute for Advanced Research, and the Canadian Foundation for Innovation. FL is supported by the US Department of Energy under award number DE-FG5206NA26170. AJB, CK and ESS gratefully acknowledge the hospitality of the Kavli Institute for Theoretical Physics in Santa Barbara, where part of this work was carried out and supported by the US National Science Foundation (NSF) under grant no. PHY05-51164. All the calculations were performed using SHARCNET supercomputing facilities.

\section{References}

[1] Chakravarty S, Gelfand M P and Kivelson S 1991 Science 254970

[2] Chakravarty S and Kivelson S 1991 Europhys. Lett. 16751

[3] Chakravarty S and Kivelson S 2001 Phys. Rev. B 64064511

[4] Lin F, Šmakov J, Sørensen E S, Kallin C and Berlinsky A J 2005 Phys. Rev. B 71165436

[5] White S R, Chakravarty S, Gelfand M P and Kivelson S A 1992 Phys. Rev. B 455062

[6] Sondhi S L, Gelfand M P, Lin H Q and Campbell D K 1995 Phys. Rev. B 515943

[7] Goff W E and Phillips P 1992 Phys. Rev. B R46 603

[8] Goff W E and Phillips P 1993 Phys. Rev. B 483491

[9] Wang Z, Ke X, Zhu Z, Zhu F, Ruan M, Chen H, Huang R and Zheng L 2001 Phys. Lett. A 280351

[10] Iqbal Z et al 2003 Eur. Phys. J. B 31509

[11] Prinzbach H, Weller A, Landenberger P, Wahl F, Worth J, Scott L T, Gelmont M, Olevano D and van Issendorff B 2000 Nature $\mathbf{4 0 7} 60$

[12] Lin F, Sørensen E S, Kallin C and Berlinsky A J 2007 Phys. Rev. B 76033414 (Preprint cond-mat/0701727)

[13] Sénéchal D, Perez D and Pioro-Ladrière M 2000 Phys. Rev. Lett. 84522

[14] Sénéchal D, Perez D and Plouffe D 2002 Phys. Rev. B 66075129

[15] Ellzey M L Jr and Villagran D 2003 J. Chem. Inf. Comput. Sci. 431763

[16] Yamamoto T, Watanabe K and Watanabe S 2005 Phys. Rev. Lett. 95065501

[17] White S R, Scalapino D J, Sugar R L, Loh E Y, Gubernatis J E and Scalettar R T 1989 Phys. Rev. B 40506

[18] Zhang Y and Callaway J 1989 Phys. Rev. B 399397

[19] Hirsch J E 1983 Phys. Rev. B 284059

[20] Blankenbecler R, Scalapino D J and Sugar R L 1981 Phys. Rev. D 242278

[21] Scalettar R T, Dagotto E, Bergomi L, Jolicoeur T and Monien H 1993 Phys. Rev. B 4712316

[22] Lammert P E and Rokhsar D S 1993 Phys. Rev. B 484103 